\title{
Online Monitoring of Corrosion in a Liquid-Steam Line Pipe of the Miravalles Geothermal Field
}

\author{
G. Tres, ${ }^{1, *}$ E.A. Saborío, ${ }^{2}$ L.R. Ajún, ${ }^{3}$ A. Arias, ${ }^{4}$ A. Rodríguez, ${ }^{5}$ \\ O. Bravo, ${ }^{6}$ J.M. Malo ${ }^{7}$ \\ ${ }^{1,2}$ Centro de Investigación en Corrosión (CICorr)- Instituto Costarricense de Electricidad \\ (ICE) \\ 3,4 Ingeniería de Plantas de Potencia- Instituto Costarricense de Electricidad (ICE) \\ ${ }^{5}$ Área de Geociencias, Recursos Geotérmicos- Instituto Costarricense de Electricidad (ICE) \\ ${ }^{6}$ Centro de Electroquímica y Energía Química (CELEQ), Universidad de Costa Rica \\ ${ }^{7}$ Instituto de Investigaciones Eléctricas (IIE), México
}

Received $9^{\text {th }}$ July 2007; accepted $21^{\text {st }}$ December 2007

\begin{abstract}
The goal of this study was to evaluate the internal corrosion in a liquid-steam pipeline (type A515 low carbon steel) of the Geothermal Field of Miravalles (CGM) in Costa Rica, and to determine the corrosion thickness for future geothermal fields. The monitoring was carried out using the following techniques: linear polarization resistance (LPR), electrical resistance (ER), and weight loss. The results show for neutral or alkaline $\mathrm{pH}$ conditions and slightly free of oxygen, that the corrosion thickness should be $1.8 \mathrm{~mm}$, in order to guarantee the integrity of the pipe over the field lifetime.
\end{abstract}

Keywords: erosion, LPR, ER, gravimetric method, ultrasound.

\section{Introducción}

El Campo Geotérmico Miravalles está ubicado en las faldas del volcán Miravalles de Costa Rica y ha sido objeto de perforaciones profundas desde 1979 [1]. Se han puesto en marcha tres plantas de condensación, en 1994, 1998 y 2000, respectivamente, así como una de ciclo binario en el 2004. La capacidad instalada actualmente es de $163 \mathrm{MW}$, que representan el 8,4\% del total del país, así como un $15 \%$ de la energía que se produce. El CGM cuenta con 52 pozos profundos, de los cuales 30 son para la producción de vapor y 13 para reinyección; el resto son para respaldo operativo. La vida útil proyectada para el campo es de treinta años. [2] El transporte de los fluidos geotérmicos, tanto en fase líquida, vapor o bifásica (líquida- vapor), se realiza por medio de tuberías aéreas, de diferentes aceros al carbono, tales como: ASTM A515, ASTMA53,

\footnotetext{
* Corresponding author. E-mail address: gtres@ice.go.cr
} 
ASTM A234, ASTM A36 y API 5L. Las mismas están recubiertas externamente con una fibra de vidrio y una cubierta de aluminio, de un espesor total de 2.5 a 5 cm (1-2 pulg) y sus funciones principales son evitar la transferencia de calor al medio y proteger al metal base de agentes corrosivos atmosféricos. De esta manera, el estudio se enfocó únicamente al desgaste interno.

El diseño mecánico para los diámetros y espesores requeridos depende de variables como tipo y patrón de flujo, presión, temperatura y tipo de material; estos espesores mínimos se determinan de acuerdo con la norma ASME/ANSI B31.1.1 [3]; luego debe incluirse un sobreespesor de seguridad y finalmente otro por desgaste. Específicamente el sobreespesor por corrosión fue recomendado por una empresa comercial, sin un estudio previo; siendo $3 \mathrm{~mm}$ en tramos rectos y $5 \mathrm{~mm}$ en codos. Como es bien sabido, uno de los aspectos de mayor importancia para la selección de materiales y espesores por corrosión, consiste en determinar la velocidad de desgaste y tipo de deterioro que sufre el metal. Por esta razón, se optó por el estudio en línea y no en las condiciones de laboratorio, los cuales se realizaron a presión atmosférica y temperaturas máximas de $94{ }^{\circ} \mathrm{C}$ [4].

De hecho, el costo por sobre espesor de $1 \mathrm{~mm}$ para $30.5 \mathrm{~km}$ de tuberías, asciende a más de un millón de dólares [5]. A partir de esta investigación, se estimó una posible reducción de sobreespesor en la construcción del futuro proyecto geotérmico denominado Pailas, a desarrollarse en la misma zona geográfica a partir del año 2008. De la misma forma, se desarrollará un programa mantenimiento y reemplazo de tuberías cuya vida útil se haya alcanzado o cuya integridad pueda verse comprometida.

A través del tiempo se han utilizado diferentes métodos para estimar el desgaste por corrosión; probablemente el más conocido es el gravimétrico, o de pérdida de masa [6]; éste se ha empleado con mucho éxito por ser muy exacto pero tiene el inconveniente que requiere generalmente largos períodos para poder construir gráficas de velocidad de corrosión, además de no generar información específica sobre el mecanismo de reacción. Por otro lado, existen las técnicas electroquímicas que permiten la evaluación de la cinética y el mecanismo de reacción, realizando medidas instantáneas y teniendo una alta sensibilidad. El primer método, denominado de intersección, fue desarrollado de manera experimental por Tafel a principios del siglo XIX [7] y permite la obtención de las curvas de polarización completas pero con el inconveniente que las grandes polarizaciones, en la dirección anódica, destruyen la superficie del metal. Actualmente uno de los más populares y utilizados es el propuesto por Sterny Geary en 1957 [8] y se denomina método de Resistencia de Polarización Lineal (RPL), el cual permite realizar muchas mediciones sin que se dañe el electrodo de trabajo, ya que las polarizaciones son muy pequeñas, alrededor de $\pm 10 \mathrm{mV}$ del potencial de corrosión ( $\left.\mathrm{E}_{\text {corr }}\right)$. Finalmente, otra técnica que está cobrando gran aplicación es la de resistencia eléctrica (RE), con la cual se miden los cambios de densidad de corriente debido a la pérdida de material, o sea que permite medir las pérdidas tanto por efectos de corrosión como de erosión [9]. 


\section{Parte experimental}

Todos los sistemas para el monitoreo en línea se ubicaron en un tramo de la tubería del pozo 21, que transporta fluido bifásico a una temperatura de unos 170 ${ }^{\circ} \mathrm{C}, 600 \mathrm{KPa}$ ( 6 bars) de presión y flujo anular, según los resultados con HORF, y con condiciones químicas que se resumen el Cuadro I. En la Fig. 1 se esquematiza el tramo y las fotos de los sistemas instalados.

Cuadro I. Composición química de los fluidos condensados de la tubería del pozo productor 21, del Campo Geotérmico Miravalles.

\begin{tabular}{|c|c|c|c|c|c|c|c|c|}
\hline Fecha & $\mathbf{0 1 / 1 1 / 0 4}$ & $\mathbf{2 0 / 0 4 / 0 5}$ & \multicolumn{2}{|c|}{$\mathbf{0 4 / 0 2 / 0 6}$} & \multicolumn{2}{|c|}{$\mathbf{0 5 / 0 7 / 0 6}$} & \multicolumn{2}{|c|}{$\mathbf{0 9 / 1 0 / 0 6}$} \\
\hline Temperatura $\left({ }^{\circ} \mathrm{C}\right)$ & 98 & 98 & 98 & $170^{*}$ & 98 & 170 & 98 & $170^{*}$ \\
\hline $\mathrm{pH}$ & 7.87 & 7.77 & 7.83 & 6.96 & 7.97 & 7.03 & 7.93 & 6.93 \\
\hline Cond. $(\mu \mathrm{S} / \mathrm{cm})$ & 14950 & 15560 & 15810 & $\ldots$ & 15575 & $\ldots$ & 16375 & $\ldots$ \\
\hline $\mathrm{Na}^{+}(\mathrm{ppm})$ & 2986 & 2950 & 3138 & 2700 & 3126 & 2690 & 3036 & 2610 \\
\hline $\mathrm{Ca}^{++}(\mathrm{ppm})$ & 122 & 126 & 124 & 107 & 131 & 113 & 130 & 118 \\
\hline $\mathrm{Cl}^{-}(\mathrm{ppm})$ & 4975 & 5010 & 5069 & 4362 & 5016 & 4316 & 5095 & 4381 \\
\hline $\mathrm{SO}^{4-}(\mathrm{ppm})$ & 47 & 53 & 54 & 47 & 51 & 44 & 54 & 46 \\
\hline $\mathrm{HCO}^{3-}(\mathrm{ppm})$ & 23 & 29 & 28.0 & 19.2 & 24.50 & 21.50 & 14.85 & 14.51 \\
\hline $\mathrm{SiO}_{2}$ total $(\mathrm{ppm})$ & 542 & --- & 541 & 466 & 521 & 448 & 556 & 478 \\
\hline S.T.D. & 9530 & 9425 & 9460 & 8140 & 9650 & 8304 & 9555 & 8215 \\
\hline
\end{tabular}

* Corresponde a la composición química, recalculada a una temperatura similar a la de la tubería internamente $\left(170{ }^{\circ} \mathrm{C}\right)$. Para ello se utilizó el programa WATCH, versión 2,3 (2004). Las fechas tienen el formato Día/Mes/Año.

\section{Gravimetría}

Para la evaluación por gravimetría se colocaron testigos en un sistema de derivación, donado por la corporación japonesa New Energy Development Organization (NEDO) - ver foto a) de la Fig. 1 - considerando los lineamientos de la norma ASTM G-04 [10]. Estos testigos son rectangulares, de $5 \times 3 \mathrm{~cm}$, y se alojaron paralelos al flujo para simular las condiciones dentro de la tubería; los mismos fueron mecánicamente pulidos con lija grado 600, usando como abrasivo carburo de silicio, lavando con agua, enjuagando con etanol y acetona.

El sistema de derivación se mantuvo drenado a una velocidad aproximada de 1 $\mathrm{L} / \mathrm{min}$, sin retorno a la tubería. Se hizo limpieza química de los testigos de acuerdo con el procedimiento c.3.1 de la Tabla A1.1 de la norma ASTM G1[6]. La medición de las masas se llevó a cabo con una balanza analítica Sartorius BP 210S y las dimensiones con un micrómetro, marca Mitutoyo. Cada 5-6 meses se retiraron 2 testigos para calcular la velocidad de corrosión. Adicionalmente se evaluó la morfología de ataque con un estereomicroscopio Olympus, SZ-PT y un microscopio invertido metalográfico Olympus PME 3, de acuerdo con 1o establecido en la norma ASTM G-46 [11]. 


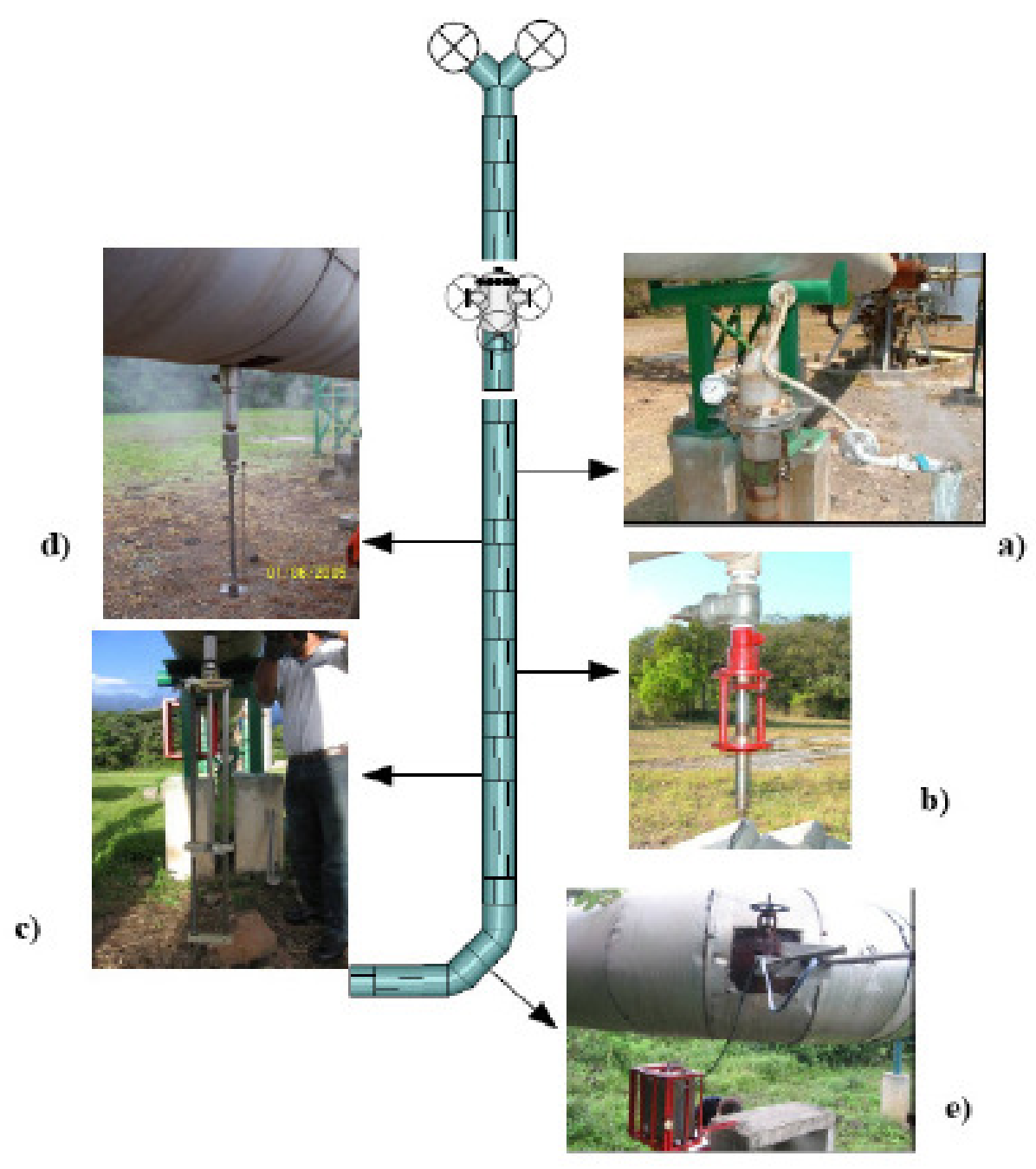

Figura 1. Esquema del tramo de la tubería del pozo 21 y fotos de las diferentes probetas empleadas en el CGM. a) Sistema de derivación para testigos por gravimetría, b) probeta para RPL, diseñado por el ICE, c) probeta marca Cormon para RPL, d) probeta marca Rohrback por resistencia eléctrica (RE) y e) probeta CEION, marca Cormon por RE.

\section{Resistencia a la polarización lineal}

Para el seguimiento de la corrosión por RPL se colocó un portaprobetas diseñado y fabricado por el ICE - ver foto b) de la Fig. 1. En la Fig. 2 se muestra un esquema y descripción del sistema; como se observa, las probetas constan de tres electrodos circulares nominalmente idénticos de acero al carbono. Con esta probeta se realizaron mediciones aproximadamente cada mes. Se empleó un potenciostato-galvanostato marca Gill AC 08, realizando corridas potenciodinámicas, polarizando $\pm 10 \mathrm{mV} \mathrm{DC}$ respecto al potencial mixto de corrosión, a una velocidad de barrido que se varió desde $3 \mathrm{mV} / \mathrm{min}$ hasta 100 $\mathrm{mV} / \mathrm{min}$. Cada vez se realizaron entre 5 a 20 lecturas, dependiendo del tiempo disponible y clima predominante, permitiendo un tiempo entre mediciones para que el electrodo de trabajo alcanzara su potencial de circuito abierto o de corrosión. También se empleó una probeta comercial marca Cormon de tres 
electrodos cilíndricos salientes de acero al carbono. Este sistema incluye un recolector de datos que permite realizar un monitoreo mucho más continuo y automatizado; los datos se tomaron y registraron cada 30 minutos. En la foto c) de la Fig. 1 se muestra el portaprobeta instalado. Los parámetros utilizados fueron los siguientes: mediciones potenciostáticas anódicas de $30 \mathrm{~s}$ y con un voltaje de polarización de $+10 \mathrm{mV}$ vs. $\mathrm{E}_{\text {corr }}$. Ambas probetas se ubicaron en la parte inferior de la tubería, para asegurar el contacto de los electrodos con el electrolito.
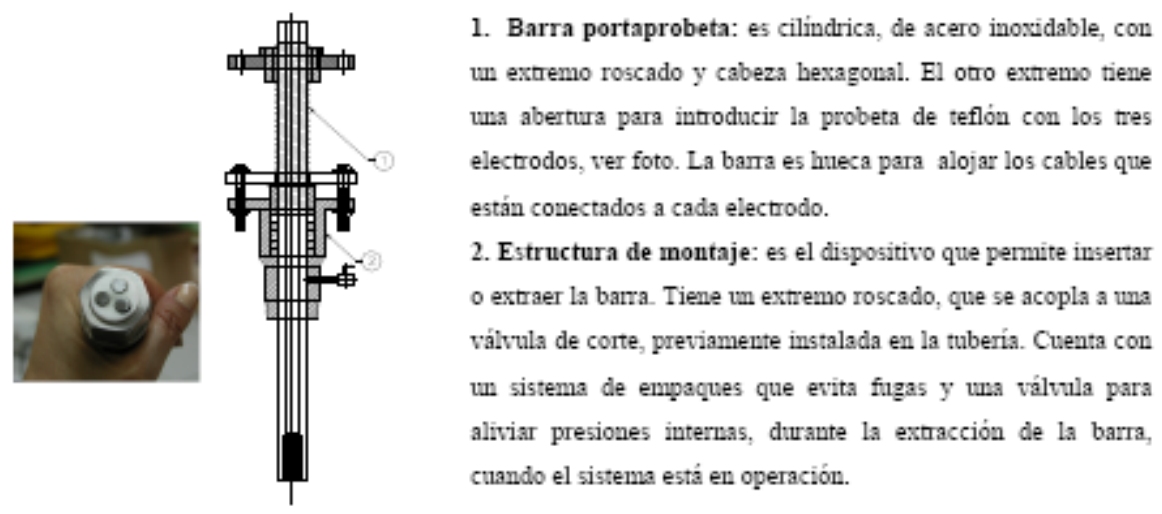

Figura 2. Esquema del portaprobetas diseñado y construido por el ICE y una fotografía de la celda, para el monitoreo en línea en el CGM, por medio de la técnica de RPL.

\section{Resistencia eléctrica}

Se utilizaron dos portaprobetas comerciales. Uno marca Rohrback, con un sensor de acero al carbono, tipo T4 saliente y espesor transversal de $0.05 \mathrm{~mm}$, cubierto con un protector de acero inoxidable para evitar al máximo el efecto erosivo. Este se colocó en la parte inferior de un tramo recto, ver foto d) de Fig. 1. La toma de datos fue manual, con el equipo Marca Rohrback, modelo CK-4, serie 0231. La segunda probeta es marca Cormon, modelo CEION, con un sensor en forma de espiral y un espesor transversal de $1 \mathrm{~mm}$, la cual se instaló en un codo, ver foto e) de la Fig. 1. El sensor es de acero al carbono y tiene un corte de $45^{\circ}$. El sistema incluye un recolector de datos, que mide y almacena datos cada 12 horas. Las probetas se instalaron de manera que los sensores quedaron inmersos en la salmuera geotérmica.

\section{Ultrasonido}

Finalmente se han medido espesores de pared en diferentes tuberías del CGM, por el método de ultrasonido, con un equipo marca Krautkrämer, modelo USD. Las mediciones se realizaron al principio cada seis meses y luego cada año, durante los paros por mantenimiento. Estas mediciones se hicieron iniciando en la parte superior de la tubería, cada $45^{\circ}$, en el sentido de las manecillas del reloj y frente a la dirección del flujo. Se hicieron mediciones en 10 puntos del CCM, ver Fig. 3 y Cuadro II. 


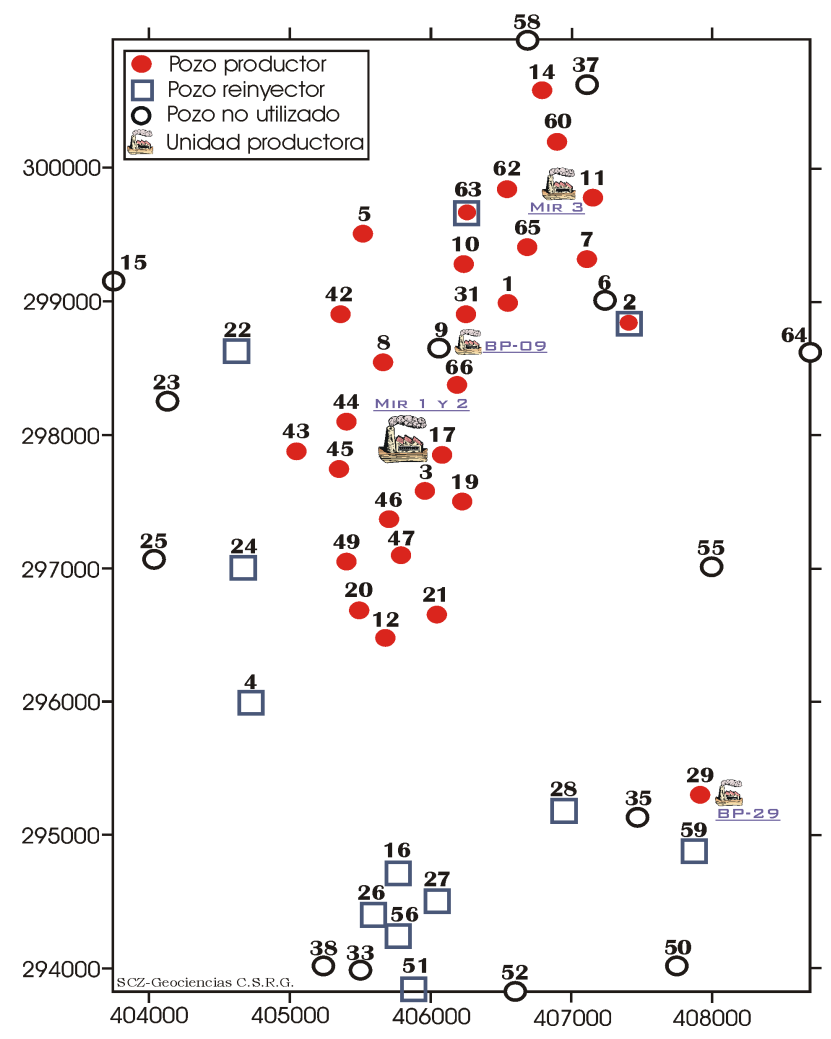

Figura 3. Representación del Campo Geotérmico Miravalles. Las mediciones de espesores por ultrasonido se hicieron en tramos de tuberías del pozo 21, 22 y 45.

Cuadro II. Puntos de medición de espesores por ultrasonido en las tuberías del Campo Geotérmico Miravalles.

\begin{tabular}{|c|c|c|c|c|c|}
\hline Tramo & $\begin{array}{c}\phi^{*} \\
(\mathrm{~mm})\end{array}$ & $\begin{array}{l}\text { Punto de } \\
\text { medición }\end{array}$ & Acero & Fluido & $\begin{array}{c}\text { Espesor nominal } \\
\text { mm } \pm 12 \%\end{array}$ \\
\hline \multirow{3}{*}{ Pozo 21} & \multirow{3}{*}{650} & $\mathrm{P} 1$ & A515 & Bifásico & 9.52 \\
\hline & & P2,codo & A234 & Bifásico & 19.6 \\
\hline & & P3 & A515 & Bifásico & 9.52 \\
\hline \multirow{2}{*}{ Sat. III - Sat. II } & \multirow{2}{*}{800} & P4,codo & A234 & Vapor & 12.7 \\
\hline & & P5 & A515 & Vapor & 12.7 \\
\hline Sat. IV, unión 14 & 500 & P6 & A515 & Vapor & 9.52 \\
\hline \multirow{2}{*}{ Pozo 22} & \multirow{2}{*}{350} & P7 & A53 & Líquido & 7.52 \\
\hline & & P8,codo & A234 & Líquido & 9.52 \\
\hline \multirow{2}{*}{ Pozo 45} & \multirow{2}{*}{1050} & P9 & API 5L & Bifásico & 12.7 \\
\hline & & P10 & API 5L & Bifásico & 12.7 \\
\hline
\end{tabular}




\section{Resultados y discusión \\ Gravimetría}

En la Fig. 4 se muestra el gráfico de velocidad de corrosión obtenido. Los resultados indican una disminución en la pérdida de masa, que para corrosión generalizada, representa un desempeño excepcionalmente bueno del metal en las condiciones de operación [12].

En la Fig. 5 se muestra la superficie, antes y después de la limpieza química de una muestra luego de extraída. Como se observa en a) y c), los productos de corrosión son oscuros y poco porosos; también durante la limpieza se constató que los mismos son adherentes. En todos los testigos el desgaste fue uniforme.

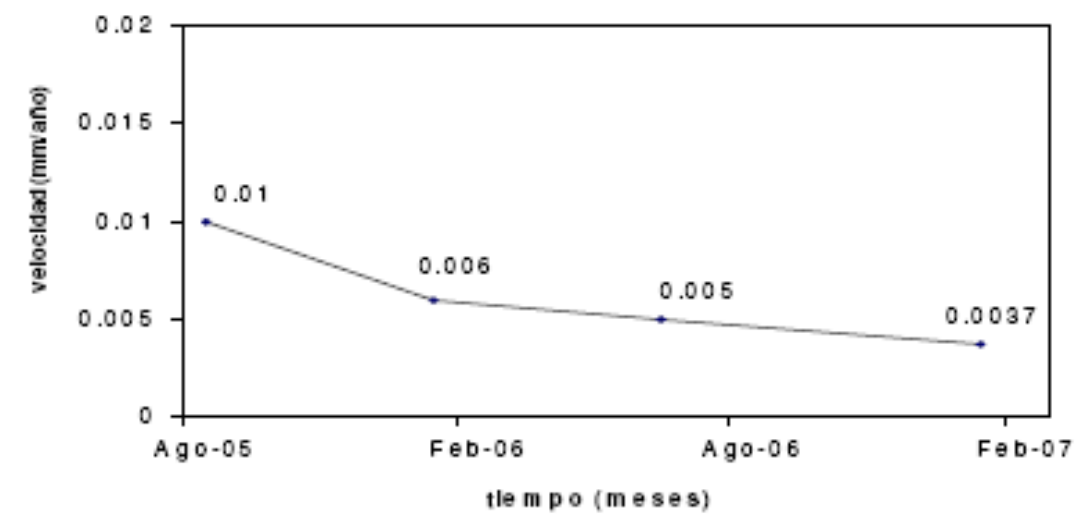

Figura 4. Velocidad de corrosión en mm/año vs. tiempo obtenidos por gravimetría.
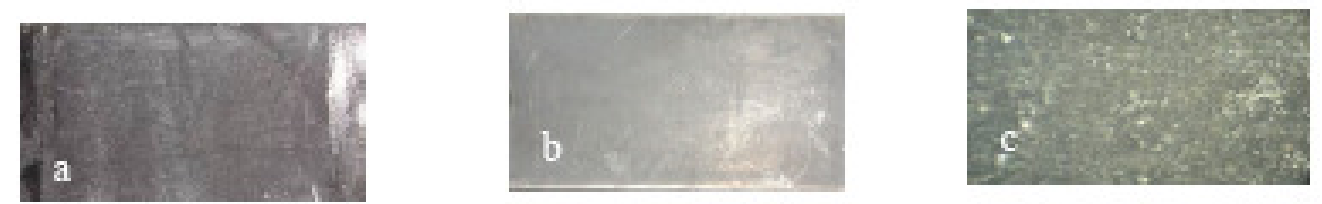

Figura 5. Fotografías digitales del testigo S5, extraído de un sistema de derivación en una tubería del pozo 21 del CGM: a) antes y b) después de la limpieza química, c) productos a $200 \mathrm{x}$.

Como se aprecia en la Fig. 4, a partir de agosto del 2006 la velocidad bajó prácticamente a la mitad, manteniéndose en el tiempo; estos resultados concuerdan con el aumento de incrustación, que puede proteger la superficie metálica de la acción electrolítica del medio [13]. En este sistema no se reprodujeron todas las condiciones de operación; particularmente la velocidad de la fase líquida, en la tubería de $1 \mathrm{~m} / \mathrm{s}$ y en la derivación de $0.1 \mathrm{~m} / \mathrm{s}$; y la temperatura, ya que no se colocó un aislante térmico; estos factores pudieron favorecer la formación de sales y velocidades de corrosión menores a las obtenidas con probetas situadas directamente en las tuberías.

\section{Resistencia de polarización lineal}

\section{A. Probeta-ICE}

Previo al monitoreo en línea se hizo un estudio de laboratorio con los diferentes aceros empleados en la construcción de las tuberías, utilizando salmueras geotérmicas ligeramente alcalinas. El mismo indicó una diferencia de 
velocidades por pérdida de masa y técnicas electroquímicas menores a un orden de magnitud, [4]. A partir de esto, se confeccionaron varios prototipos de probetas para instalar en una tubería que fallaron mecánicamente hasta obtener una adecuada, que se muestra en las Fig. 1b) y 2). Para las cálculos de las velocidades por RPL, se utilizó el valor intermedio de $\mathrm{B}=0.026 \mathrm{~V}$, debido a que esta constante, en la ecuación de Stern y Geary, varía poco con los valores de las pendientes de Tafel, ya que aparecen tanto en el numerador como en el denominador $\left(B=b_{a} * b_{c} / 2.3 *\left(b_{a}+b_{c}\right)[6]\right.$. Los resultados se muestran en la Fig. 6. Con esta misma probeta se midieron, las variaciones naturales en corriente y voltaje entre el electrodo de trabajo y el de referencia, por medio del método de ruido electroquímico [14]. Sin embargo, los valores de resistencia en ruido $\left(\mathrm{R}_{\mathrm{r}}\right)$ no fueron reproducibles con los datos de RPL, que es lo propuesto por el método [15].

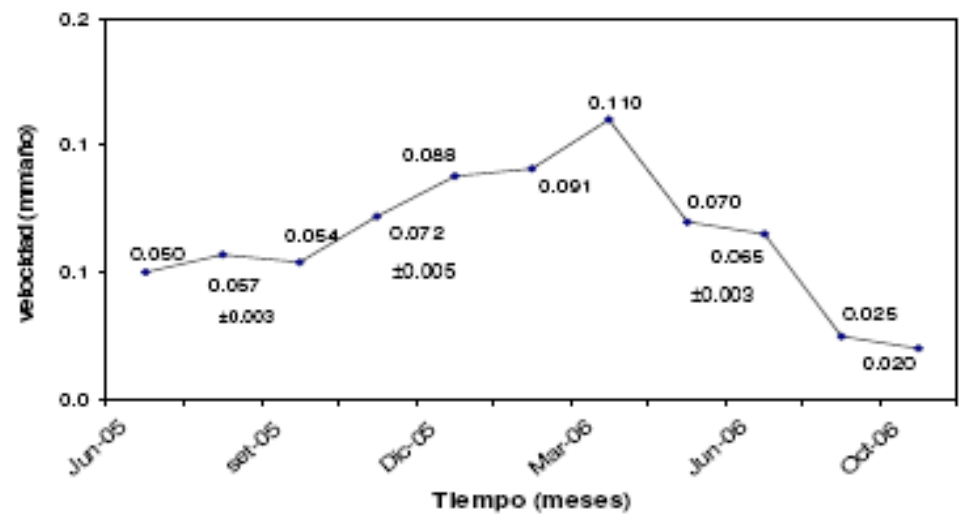

Figura 6. Velocidad de corrosión en mm/año vs. tiempo, por RPL. Sistema ICE.

Los valores de los primeros meses mayores a $0.2 \mathrm{~mm} / \mathrm{año}$ se debieron a desajustes del potenciostato, según se corroboró con los procedimientos de verificación de los circuitos, descritos en ASTM G59 [16] y ASTM G 106 [17], por lo cual no se tomaron en cuenta para el análisis. De esta manera, desde junio del 2005 se usó un equipo debidamente calibrado y las velocidades y variaciones en el tiempo resultaron mucho menores, ver la Fig. 5. En esta figura se reporta la incertidumbre de algunos resultados, con el fin de ilustrar la precisión de los mismos. El promedio fue de $0.06 \mathrm{~mm} /$ año y coincide, en orden de magnitud, con el primer dato de gravimetría de la Fig. 3; esto probablemente porque los primeros testigos tenían pocas incrustaciones y el proceso de corrosión no estaba siendo enmascarado por las mismas.

Según se observa en la Fig. 7, los electrodos de la probeta no se incrustaron pero los testigos por gravimetría sí; además con el tiempo el grado de incrustaciones. Las mediciones de RPL se realizaron mensual o bimensualmente, debido a que no se contó con un registrador de datos en línea y había que transportar el potenciostato unos $200 \mathrm{~km}$ al CGM. Esto explica que se obtuvieran pocos datos con una técnica que permite realizar mediciones instantáneas de la corrosión. Probablemente la mayor ventaja de utilizar un equipo con un "software" versátil es que se pueden variar las condiciones de ensayo e incluso aplicar otras técnicas electroquímicas de manera secuencial, tal como se hizo en este estudio. Así 
mismo, al poder variar la velocidad de barrido, se corroboró que el sistema siempre alcanzó el estado estacionario sin que se afectara el cálculo de Rp, [7]. Por otro lado, las velocidades corresponden a un promedio de mediciones, lo que permite establecer la precisión de cada dato. Finalmente, las resistencias de polarización se calcularon a partir de las gráficas de densidad de corriente vs. potencial para verificar los resultados del equipo.

a)

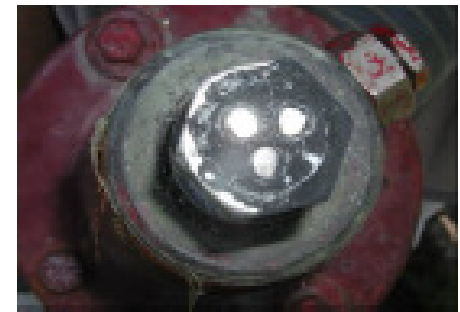

b)

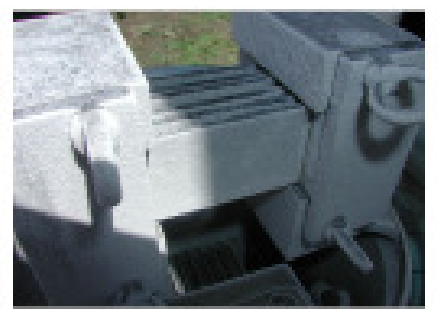

Figura 7. a) Estado de electrodos en probeta ICE-Rp, sin incrustaciones o daño localizado y b) testigos en sistema de derivación, ambos a los 6 meses de exposición.

Luego del año de exposición de la probeta, se realizó una curva de polarización, a una velocidad de $0.5 \mathrm{mV} / \mathrm{min}$, ver Fig. 8. El corrimiento que se observa entre las curvas, se debe a que el barrido anódico se hizo cuando aún no se había alcanzado el potencial de circuito abierto. El valor cercano a cero del $\mathrm{E}_{\text {corr }}$ se debe a que la celda consta de electrodos del mismo metal. Lo más importante de destacar es que la velocidad de corrosión calculada por Tafel, a partir de la curva catódica, resultó en $0.056 \mathrm{~mm} / \mathrm{año}$; lo cual es totalmente concordante con el promedio obtenido por RPL.

El gráfico de la Fig. 8 indica un control mixto de la cinética, es decir ambas hemireacciones contribuyen significativamente a la velocidad global del proceso. De ser posible este ensayo se repetirá en el campo para corroborar el mecanismo en las condiciones de operación.

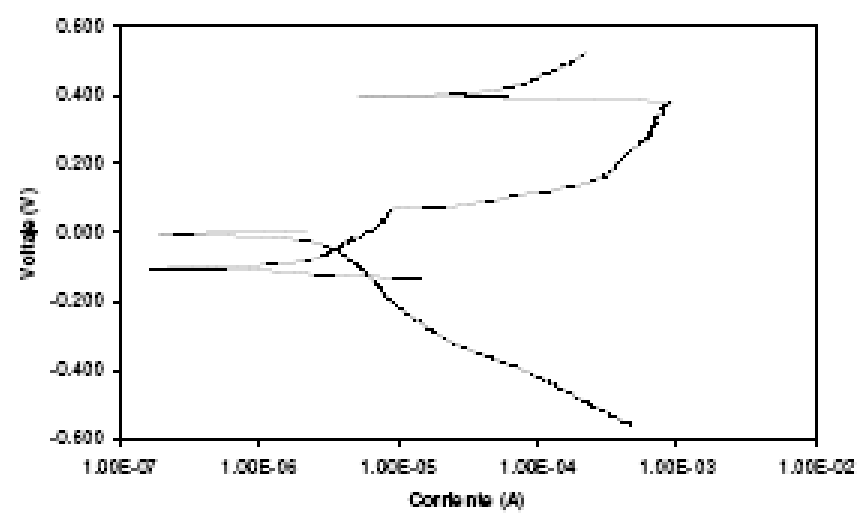

Figura 8. Curva de polarización, voltaje vs. corriente, después de un año de exposición. 


\section{B. Sistema-comercial, marca Cormon}

Este sistema es automatizado, con medición y registro de datos cada media hora, lo cual permite obtener mayor información a menores costos [18]. Como se aprecia en el gráfico de la Fig. 9, desde diciembre-2005 hasta agosto-2006, la velocidad se mantuvo generalmente por debajo de $0.1 \mathrm{~mm} /$ año, para un promedio de $0.06 \mathrm{~mm} / \mathrm{año}$, que coincide con el de la probeta-ICE. Esto sugiere, que la geometría de los electrodos no influyó, en estas condiciones de operación. Por otro lado, los valores más altos al inicio, obedecen al desgaste típico de los metales cuando no se han formado productos que protegen contra la corrosión.

La línea de tendencia que se muestra en cada uno de los tres periodos del gráfico de la Fig. 8, corresponden a ecuaciones polinomiales de grado cuatro, que se indican a continuación:

Primer período: $y=3^{-8} x^{4}-4^{-3} x^{3}+2.3^{2} x^{2}-6^{6} x+6^{10}$

Segundo período: $\mathrm{y}=1^{-8} \mathrm{x}^{4}-1.9^{-3} \mathrm{x}^{3}+1.12^{2} \mathrm{x}^{2}-3^{6} \mathrm{x}+3^{10}$

Tercer período: $y=-2^{-7} x^{4}+2.7^{-2} x^{3}-1.6^{3} x^{2}+4^{7} x-4^{11}$

$$
\begin{aligned}
& \mathrm{R}^{2}=0.6273 \\
& \mathrm{R}^{2}=0.7658 \\
& \mathrm{R}^{2}=0.5322
\end{aligned}
$$

No se logró mejorar el coeficiente de correlación $\mathrm{R}^{2}$, empleando polinomios de grado mayor.

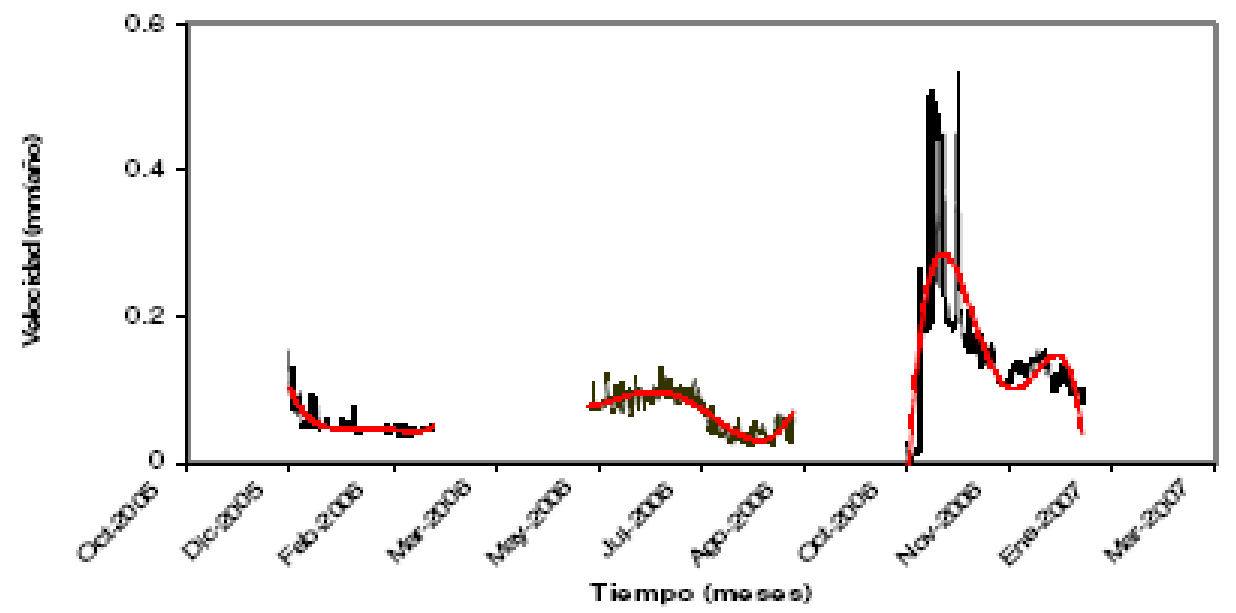

Figura 9. Velocidad de corrosión, en mm/año vs. tiempo, por RPL. Sistema comercial, Cormon.

Debido a que se recolectaron más de 4500 datos se aplicó un análisis estadístico, empleando el programa Minitab 14.20 y de acuerdo con lo establecido en la norma ASTM G16 [19]. Se encontró que el mejor ajuste corresponde a una función logarítmica normal, con $\sigma=0.5$. En la Fig. 10, se compara con la distribución normal a) para efectos de comparación. 

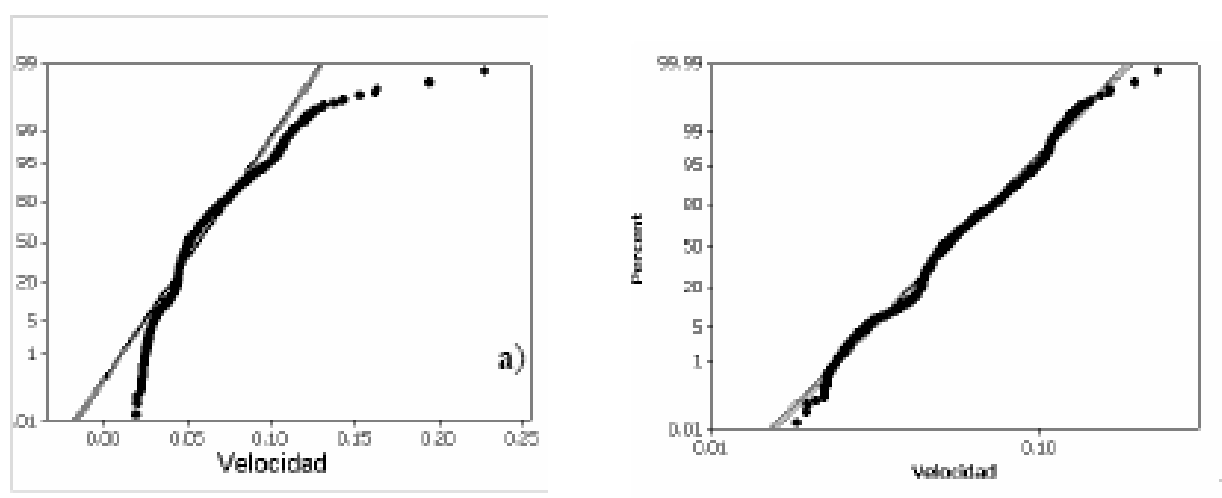

Figura 10. Ajuste de datos de 9 meses de monitoreo con la probeta de Rp-Cormon, porcentaje vs. velocidad, a) en una distribución normal y b) en una curva logarítmica normal, ambas al 95\% de confianza.

Los electrodos no sufrieron un desgaste preferencial en los extremos más cercanos a la superficie de la tubería, donde teóricamente hay mayor velocidad del fluido en fase líquida y cantidad de electrolito. Cuando se extrajo la probeta luego de un año, durante el paro por mantenimiento, se corroboró que no contenía sales depositadas y que el desgaste fue homogéneo en toda el área expuesta. La probeta se instaló nuevamente y como se advierte en la Fig. 8, la velocidad aumentó. Esto puede explicarse, por la entrada de aire durante la despresurización de la línea. Sin embargo, el paro fue del 9 al 16 de octubre2006 y se desconoce porque este efecto se mantuvo por más de 2 meses. Se decidió hacer el análisis, sin incluir estos datos, debido a que con las otras técnicas no se observó dicho efecto; por ejemplo la lectura manual de resistencia de polarización correspondiente al paro fue de $0.020 \mathrm{~mm} / \mathrm{año}$, tal y como se muestra en la Fig. 6; además por resistencia eléctrica con la probeta CEION se detectó un aumento en el desgaste, únicamente durante el paro, como se explica en el siguiente apartado. Para determinar con mayor exactitud si el efecto de los paros en el aumento de la velocidad de corrosión es significativo con respecto al resto del año, es necesario monitorear al menos durante 2 años continuos.

\section{Resistencia eléctrica}

\section{A. Probeta comercial, marca Rohrback}

Las mediciones se realizaron durante 300 días, al inicio diariamente y luego se determinó con una periodicidad menor para asegurar datos por encima del límite de cuantificación del equipo; esto es $1 \%$ del espesor transversal que corresponde a $0.000508 \mathrm{~mm},[20]$. Actualmente el mismo modelo se distribuye con límite de $0.05 \%$.

En el gráfico de la Fig. 11 se muestra la pérdida de material. Para estimar la velocidad se calculó la pendiente entre los puntos extremos de cada una de las rectas. Este gráfico muestra al inicio un pequeño aumento del desgaste, típico de materiales desnudos en un medio electrolítico; para luego estabilizarse cuando se forman productos, en algún grado protectores. El aumento a $0.06 \mathrm{~mm} / \mathrm{año}$ calculado entre Agosto y Octubre del 2005, puede estar relacionado con efectos simultáneos de corrosión y erosión; aunque teóricamente este sensor sólo debería 
mostrar desgaste por corrosión, ya que tiene una cubierta de acero inoxidable que lo protege. Entre Septiembre y Noviembre se hicieron pocas mediciones y la pendiente indica un desgaste de $0.02 \mathrm{~mm} / \mathrm{año}$. Lo más destacable es que los valores más altos coinciden en orden de magnitud con los de la técnica electroquímica; lo cual sugiere que en este tramo recto, el efecto de la erosión por impacto no es significativo. Durante los últimos cinco meses, los resultados mostraron una reducción en la resistencia eléctrica, lo cual puede deberse a la formación de productos de corrosión insoluble y conductores, tal como el sulfuro de hierro, FeS [21]; o como ocurrió en este caso, donde el sensor y la cubierta se dañaron según se comprobó cuando se extrajo la probeta en Mayo.

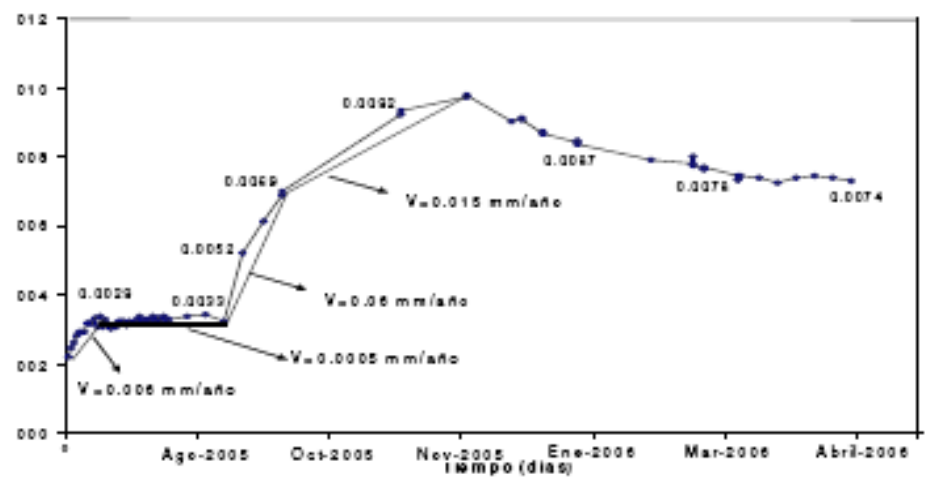

Figura 11. Desgaste de la tubería por erosión, expresada en pérdida de metal en mm vs. tiempo. Sistema comercial, Rohrback.

\section{B. Probeta comercial CEION, marca Cormon}

Con este sistema se esperaba obtener los desgastes mayores; en primer lugar porque con el sensor de acero al carbono, es posible medir simultáneamente pérdidas por erosión y corrosión. Además, porque en codos el desgaste tiende a ser mayor, con respecto a tramos rectos, debido a la aceleración del flujo por el cambio de dirección. Sin embargo, como se aprecia en la Fig. 12, la mayoría de los datos oscilaron alrededor de cero mm/año. Estos resultados no se deben al límite de cuantificación (0.0004\% del espesor transversal del sensor) [22], ya que este sistema es mucho más sensible que el marca Rorhback con el cual se obtuvieron datos cuantificables. Un factor que pudo influir fue la colocación de la probeta, ya que como se ve en la Fig. 13 debió ser en un tramo recto y no en un codo como se hizo, pues el sensor tiene un corte de $45^{\circ}$. Por otro lado, el flujo en este tramo es muy bajo, $1 \mathrm{~m} / \mathrm{s}$ el líquido y $15 \mathrm{~m} / \mathrm{s}$ el gas, así que el efecto por cambio de velocidad en el codo puede ser despreciable [22, 23].

El caso de erosión por partículas también pudo ser imperceptible ya que, según se muestra en el Cuadro I, este pozo tiene una salinidad muy baja, por ejemplo comparada con las salmueras geotérmicas hipersalinas de las Minas del Valle Imperial de California; donde la interacción de componentes presentes, incluyendo sílice en saturación, posibilitan la formación de incrustaciones complejas en las superficies del metal expuestas a la salmuera [25] y por lo tanto de partículas sólidas en el fluido; lo que no ocurre en este caso. Además en este pozo, no se han detectado secuencias de rocas poco estables o una zona muy 
fracturada donde se desprendan detritos, que explicarían un efecto erosivo por partículas sólidas [1].

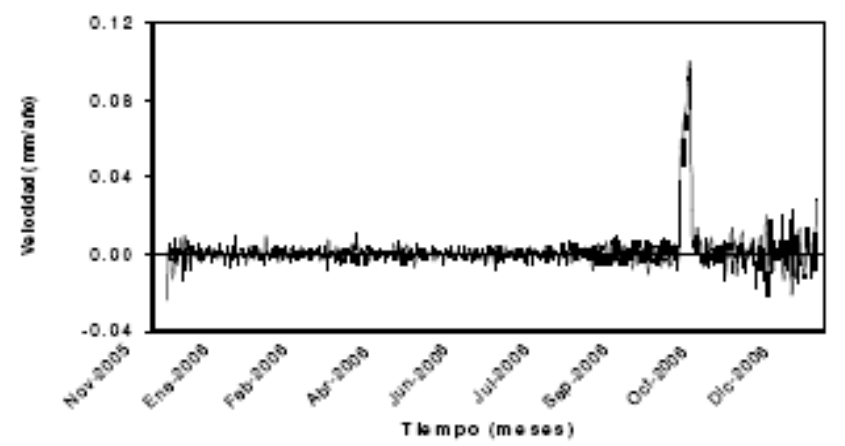

Figura 12. Desgaste en codo expresada en velocidad mm/año vs. tiempo, obtenida por RE. Sistema comercial, CEION.

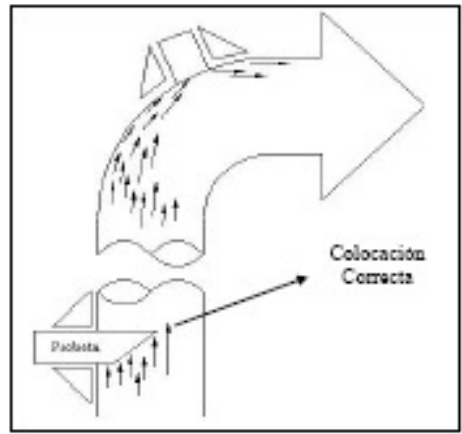

Figura 13. Forma y colocación correcta de un sensor, para evaluar pérdidas de metal por la técnica de resistencia eléctrica en una tubería.

Igualmente, no se detectaron pérdidas por corrosión, lo cual puede deberse a capas de electrolito muy delgadas o incluso ausentes, ya que en el codo la fase líquida es fluctuante por efecto de la turbulencia. Debido a que se trata de un flujo bifásico, no es posible saber cuál es el espesor de la capa de electrolito en el sensor; sin embargo, los resultados indican una pérdida insignificante debido a fenómenos electroquímicos.

En el mes de octubre se produce un aumento considerable del desgaste, coincidente con el paro por mantenimiento. Lo cual se explica por a la entrada de oxígeno al sistema, que incrementa la velocidad de corrosión hasta un valor máximo aproximado de $0.1 \mathrm{~mm} /$ año. Incluir estos datos, por ejemplo con los más de 4500 datos de RPL, no afectan el promedio de $0.06 \mathrm{~mm} / \mathrm{año}$.

\section{Ultrasonido}

Esta técnica funcionó como una excelente herramienta para corroborar que el desgaste en tuberías que transportan fluidos con $\mathrm{pH}$ ligeramente alcalino es menor a $3 \mathrm{~mm}$ para los treinta años de vida útil estimados; además no se determinó en ningún punto de medición un desgaste preferencial, ver Fig. 14. Sin embargo, el ultrasonido no es adecuado para estimar el desgaste en este caso 
específico, donde la velocidad de corrosión no es suficientemente alta como para que la sensibilidad de la técnica permita diferenciar la pérdida de metal entre una medición y otra; además la colocación del palpador en la misma posición es muy difícil de lograr, lo que genera un error indeterminado.

Finalmente, unas mediciones se hicieron en caliente y otras a temperatura ambiente durante los paros de mantenimiento y aunque se utilizó un palpador resistente a las temperaturas de operación $\left(160{ }^{\circ} \mathrm{C}-170^{\circ} \mathrm{C}\right)$, se considera que esto pudo introducir errores en los resultados, que no se pueden determinar.

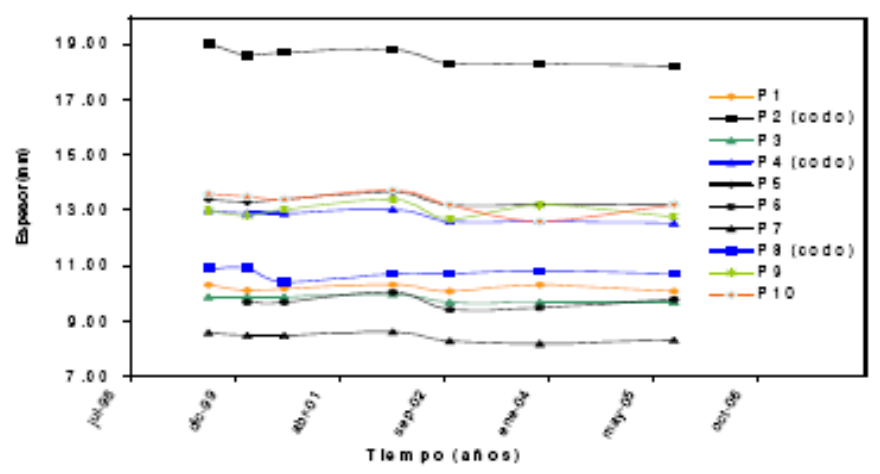

Figura 14. Medición de espesores por ultrasonido vs. tiempo, en diferentes tramos del CGM.

\section{Conclusiones y recomendaciones}

Este fue el primer estudio de la corrosión en línea de Costa Rica, utilizando tres diferentes técnicas y durante más de dos años; el cual, además se complementó con mediciones de espesores por ultrasonido por seis años.

Una de las principales conclusiones fue que el desgaste, en tuberías con fluido bifásico, se produce de manera homogénea, por lo cual se pueden utilizar los datos para definir las especificaciones de sobreespesor por corrosión.

A partir del análisis de los resultados, por los diferentes métodos, se sugiere utilizar $0.06 \mathrm{~mm} / \mathrm{año}$ para calcular el sobreespesor por corrosión-erosión, en tuberías que transporten fluidos ligeramente alcalinos y de baja salinidad en el nuevo proyecto geotérmico Pailas; así como para la sustitución de tramos en el campo de Miravalles.

Con el fin de obtener datos más confiables, se sugiere utilizar sistemas con retorno a la tubería original y así mantener todas las condiciones del sistema en operación, que como se observó en este estudio afectó los resultados obtenidos por gravimetría.

Se recomienda colocar la probeta comercial, marca Cormon-Ceion en un tramo recto, donde se presuma o conozca hay efectos erosivos, para determinar si en estos casos se debería recomendar otro valor para el cálculo de sobreespesor por desgaste. 


\section{Agradecimientos}

Se agradece el aporte del Ministerio de Ciencia y Tecnología (MICIT) para la presentación de este estudio en El XIV Encuentro de la Sociedad Portuguesa de Electroquímica.

\section{Monitoreo en Línea de la Corrosión en Tuberías de Flujo Bifásico en el Campo Geotérmico Miravalles}

\section{Referencias}

1. E. Sánchez, O. Vallejos, A. Rodríguez, et al., Chemical treatments of fluids on the Miravalles Geothermal Field, Proceedings of the World Geothermal Congress. 2005, Antalaya, Tukey, CD, 7pp.

2. A. Mainieri, Costa Rica Country updates report, Proceedings of the World Geothermal Congress, 2005, Antalaya, Tukey, CD, 5pp.

3. Power Piping ASME Code for Pressure Piping, B31, ASME B31.1, The American Society of Mechanical Engineer, New York, 2004.

4. G. Tres, E.A. Saborío, Electrochemical Evaluation of Pipelines Materials of Miravalles Geothermal Field, Congreso Iberoamericano de Corrosión y Protección, 1998, Cartagena, Colombia.

5. Ingeniería de Plantas de Potencia, UEN Proyectos y Servicios Asociados, Instituto Costarricense de Electricidad.

6. Standard Practice for Preparing, Cleaning, and Evaluating Corrosion Test Specimens, ASTM G 1-90, Vol. 03.02, 1993.

7. J.A. Gonzalez, Control de la Corrosión: Estudio y Medida por Técnicas Electroquímicas, Grafipren, S.A., Madrid, 1989. Capítulos V, VII y X.

8. $\quad$ M. Stern, A.L. Geary, J. Electrochem. Soc. 104 (1957) 56.

9. Standard Guide for On-Line Monitoring of Corrosion in Plant Equipment (Electrical and Electrochemical Methods), ASTM G 96, Vol 03.02, 1990.

10. Standard Guide for Conducting Corrosion Coupon Tests in Field Applications, ASTM G 04, Vol. 03.02, 1995.

11. Standard Guide for Examination an Evaluation of Pitting Corrosion, ASTM G 46, Vol. 03.02, 1994.

12. M.G. Fontana, Corrosion Engineering, Third Edition, McGraw Hill, 1986, United States of America, p. 172.

13. S.D. Cramer, P.B. Needham, Linear Polarization Measurements at High Temperatures in Hypersaline Geothermal Brines, Bureau of Mines Report of Investigations, 1978.

14. R.A. Cottis, S. Turgoose, J. Mendoza, The Effects of Solution Resistance on Electrochemical Noise Resistance Measurements, ASTM STP 1277, 1996, p. 93-100.

15. J.L. Dawson, Electrochemical Noise Measurement: The Definitive In-Situ Technique for Corrosion Applications, ASTM STP 1277, 1996, p. 3-35. 
16. Standard Test Method for Conducting Potentiodynamic Polarization Resistance Measurements, ASTM G 59, Vol. 03.02, 1997.

17. Standard Practice for Verification of Algorithm and Equipment for Electrochemical Impedance Measurements, ASTM G 106, Vol. 03.02, 1999.

18. G.A. Jensen, D.W. Shannon, et al., Real time Sensors in Geothermal Fluids, Their Costs and Benefits, Canadian Western Region Meeting of the National Association of Corrosion Engineers, 1982.

19. Standard Guide for Applying Statistics to Analysis of Corrosion Data, ASTM G 16, Vol. 03.02, 1995.

20. http://www.rohrbackcosasco.com/datasheets/products/CK-4.pdf. 2007.

21. Standard Guide for On-Line Monitoring of Corrosion in Plant Equipment, ASTM G 96, Vol. 03.02, 1996.

22. I.M. Hutchings, Monograph on the Erosion of Materials by Solid Particle Impact, Materials Technology of the Chemical Process Industries, Inc., Publication No.10, 1993.

23. http://www.cormon.com/products/datasheets/CMEP026.pdf. 2007.

24. http://www.cormon.com/products/datasheets/GL025_CEION.pdf. 2007.

25. J. Harrar, R. McCright, A. Goldberg, Field Electrochemical Measurements of Corrosion Characteristics of Materials in Hipersaline Geothermal Brines, Report UCRL-52376, California, 1977.

26. J. Urruchurtu, J.M. Malo, Técnicas de Monitoreo y Control de la Corrosión, IMICORR, segunda edición, 1997.

27. M.J. Danielson, Geothermal Scaling and Corrosion, ASTM STP 717, New Orleans, 1980. p. 41-56.

28. D. Shannon, Corrosion of Iron-Base Alloys versus Alternative Materials in Geothermal Brines, Report PNL-2456, Battelle Pacific Northwest Laboratories, 1977. 